\title{
Emulsion Polymerization of Chloroprene. Polymerization Mechanism and Evaluation of Crosslinking Density
}

\author{
Kenji Itoyama, ${ }^{*}$ Nobuhiro Hirashima, ${ }^{* *}$ Junji Hirano, ${ }^{* *}$ \\ and Takashi KADOWAKI*** \\ * Denka Techno-Research Co., Ltd., 1-3-D-6, Nakase, Chiba 260, Japan \\ ** Denki Kagaku Kogyo Co., Ltd., 4-1, Yurakucho I-chome, Chiyoda-ku, Tokyo 100, Japan \\ *** CRK Co., Ltd., 306, Koyagicho, Takasaki, Gunma 370, Japan
}

(Received September 12, 1990)

\begin{abstract}
The polymerization and crosslinking reaction mechanisms were studied for the emulsion polymerization of chloroprene. The dependence of volume of monomer droplet, surface-tension and crosslinking density on conversion was confirmed to be corresponding to the Harkins and Smith-Ewart models. Crosslinking densities were estimated from swelling ratios of the gel polymers prepared at various temperatures in the absence of a chain transfer reagent. The ratios of the rate constant of the propagation to the crosslinking $K$ were calculated to be $2.0 \times 10^{-4}$, $7.1 \times 10^{-4}$, and $9.9 \times 10^{-4}$ at 10,40 , and $55^{\circ} \mathrm{C}$, respectively. The difference between activation energies of these two mechanisms was $27.8 \mathrm{~kJ} \mathrm{~mol}^{-1}$. The proportions of polymer in the growing polymer particles during the existence of monomer droplets $\theta^{\prime}$ were also caluculated to be 0.47 , 0.45 , and 0.42 , at each temperature.
\end{abstract}

KEY WORDS Chloroprene / Emulsion Polymerization / Mechanism / Crosslinking / Swelling / Rate Constant / Activation Energy /

Since the development of chloroprene polymerization by Carothers et al. ${ }^{1}$ in 1931, many investigators ${ }^{2-10}$ have studied the mechanism of polymerization of chloroprene. The emulsion polymerization method has been widely employed for the production of commercially available polychloroprenes. Comapred with the relatively well understood styrene polymerization, the mechanism of the emulsion polymerization of chloroprene is less clear. $^{6}$

The purpose of this paper is to study the polymerization and crosslinking mechanism quantitatively.

Harkins ${ }^{11}$ has proposed a novel mechanism of emulsion polymerization in order to explain previous studies. Smith and Ewart ${ }^{12}$ developed quantitative kinetics and applied them to a styrene-butadiene system, taking into account that the concentration of polymer in the growing polymer particle is not always equal to the conversion in emulsion polymerization.

Flory ${ }^{13}$ has proposed that the crosslinking in diene polymerization is described by the following equation:

$$
\rho=-K[1+(1 / \theta) \ln (1-\theta)]
$$

where $\rho$ is the density of crosslinked units in the polymer, $K$ is $2 k_{\mathrm{b}} / k_{\mathrm{p}}, k_{\mathrm{b}}$, and $k_{\mathrm{p}}$ are the rate constants of crosslinking and propagation, respectively, and $\theta$ is the conversion.

Equation 1 can be extended to an emulsion polymerization system, taking into account the above-mentioned inequality. Morton and Salatiello $^{14}$ have studied the emulsion polymerization of butadiene and indirectly determined the magnitude of $K$ from gel point, chain transfer reagent concentration and polymerization rate data.

In this paper, the authors will clarify the 
Table I. Crosslinking characteristics with conversion

\begin{tabular}{|c|c|c|c|c|c|}
\hline $\begin{array}{c}\text { Polym. } \\
\text { temp. }\end{array}$ & $\begin{array}{c}\text { Sample } \\
\text { No. }\end{array}$ & $\begin{array}{c}\begin{array}{c}\text { Conver- } \\
\text { sion }\end{array} \\
\%\end{array}$ & $\begin{array}{c}\begin{array}{c}\text { Soluble } \\
\text { fraction }\end{array} \\
\%\end{array}$ & $\begin{array}{l}\text { Swell- } \\
\text { ing } \\
\text { ratio } \\
\alpha\end{array}$ & $\begin{array}{c}\text { Crosslink- } \\
\text { ing } \\
\text { density } \\
\rho \times 10^{5}\end{array}$ \\
\hline \multirow{11}{*}{$40^{\circ} \mathrm{C}$} & A & 6.33 & 13.22 & 36.09 & 4.07 \\
\hline & B & $12 / 78$ & 6.09 & 21.24 & 10.09 \\
\hline & $\mathrm{C}$ & 21.07 & 3.13 & 18.08 & 13.59 \\
\hline & $\mathrm{D}$ & 33.00 & 1.10 & 14.14 & 20.81 \\
\hline & $\mathrm{E}$ & 41.65 & 0.94 & 13.87 & 21.24 \\
\hline & $\mathrm{F}$ & 50.80 & 0.94 & 12.53 & 25.05 \\
\hline & G & 61.20 & 0.70 & 9.56 & 41.60 \\
\hline & $\mathrm{H}$ & 67.80 & 0.29 & 8.66 & 53.28 \\
\hline & I & 79.70 & 0.28 & 7.12 & 72.57 \\
\hline & $\mathbf{J}$ & 91.50 & 0.08 & 5.33 & 136.84 \\
\hline & $\mathrm{K}$ & 96.70 & & 4.12 & 240.51 \\
\hline \multirow{6}{*}{$10^{\circ} \mathrm{C}$} & A & 6.95 & 13.71 & 73.11 & 1.41 \\
\hline & B & 31.30 & 2.40 & 27.67 & 6.20 \\
\hline & $\mathrm{C}$ & 62.20 & 1.10 & 20.44 & 10.43 \\
\hline & D & 74.80 & 1.29 & 16.26 & 15.74 \\
\hline & $\mathrm{E}$ & 86.50 & 0.91 & 11.63 & 28.75 \\
\hline & $\mathrm{F}$ & 92.26 & 0.86 & 9.07 & 46.45 \\
\hline \multirow{8}{*}{$55^{\circ} \mathrm{C}$} & A & 7.20 & 3.10 & 22.37 & 7.20 \\
\hline & B & 22.20 & 1.16 & 17.22 & 18.20 \\
\hline & $\mathrm{C}$ & 30.10 & 2.36 & 13.81 & 23.50 \\
\hline & D & 38.30 & 1.50 & 12.80 & 28.20 \\
\hline & $\mathrm{E}$ & 48.70 & 1.95 & 11.95 & 34.20 \\
\hline & $\mathrm{F}$ & 56.00 & 1.31 & 9.32 & 45.80 \\
\hline & G & 67.80 & 2.96 & 7.60 & 61.00 \\
\hline & $\mathrm{H}$ & 75.10 & 0.76 & 5.96 & 82.30 \\
\hline
\end{tabular}

crosslinking reaction mechanism in the emulsion polymerization of chloroprene from measurements of the crosslinking density of the polymer $(\rho)$ prepared at various conditions in the absence of a chain transfer reagent. Ratios of rate constants of the propagation to the crosslinking, the difference in activation energies between two mechanisms and the concentration of polymer in the growing polymer 'particle can be calculated, using equations derived by a modification of eq 1 from the dependence of the crosslinking density on conversion.

\section{EXPERIMENTAL}

\section{Polymerization}

Polymerizations were carried out in 2 liter flasks. The flasks were flushed with nitrogen and placed in a waterbath. After heating to the required temperature, the persulfate solution was added gradually in order to maintain a constant polymerization rate. The flasks were rotated horizontally at 150 revolutions per minute.

Conversion data were calculated based on solids content. The recipes used were as follows;

$\begin{array}{lc}\text { chloroprene } & 100 \text { weight parts } \\ \text { water } & 150 \\ \text { rosin acid } & 3.0 \\ \text { sodium hydroxide } & 0.8 \\ \beta \text {-naphthalene sulfonic } & 0.8 \\ \quad \begin{array}{l}\text { acid performaldehyde } \\ \text { condensation sodium }\end{array} & \\ \quad \text { salt } & \\ n \text {-dodecylmercaptan } & \text { none (or variable) } \\ \text { potassium persulfate, } & \text { variable } \\ 2 \% \text { aq. soln. } & \end{array}$

\section{Materials}

Chloroprene monomer of $99.7 \%$ purity was washed with water and then distilled under reduced nitrogen pressure. The water used in the polymerization was purified by an ionexchanger and distilled. Rosin acid was purchased from Arakawa Chemical Corp. and used as received. $\beta$-Naphthalene sulfonic acid performaldehyde condensation sodium salt was a form of emulsifier ' $\mathrm{N}-1$ ' of Kao Corp. Sodium hydroxide, $n$-dodecylmercaptan, and persulfate were reagent grades purchased from Wako Chemical Corp.

\section{Determination of Swelling Ratio and Cross- linking Density \\ $20 \mathrm{~cm}^{3}$ of the polymerization mixture in latex form was sampled at the required time. In order to separate the polymer from the latex, about $50 \mathrm{~cm}^{3}$ of methanol was added. The polymer}


was reprecipitated 3 times with benzenemethanol. The final precipitate was dissolved in benzene again and freeze-dried. The purified polymer was maintained in toluene for 24 hours at $25^{\circ} \mathrm{C}$, and the swollen polymer was weighed. Then, the swollen polymer was dried under reduced nitrogen pressure, and the dried polymer was weighed.

The gel content in polymer $G$ and the swelling ratio $\alpha$ were calculated as follows:

$$
G=\left(W_{3} / W_{1}\right) \times 100(\%), \quad \alpha=\left(W_{2}-W_{3}\right) / W_{3}
$$

where $W_{1}$ is the weight of the sample before swelling, $W_{2}$ is the weight after swelling, and $W_{3}$ is the weight of the polymer after drying. The soluble fraction of the polymer equals to $(100-G),(\%)$.

By using the obtained $\alpha$-value, crosslinking density $\rho$ is calculated according to Flory's equation ${ }^{13}$ : (Primary molecular weight was assumed to be infinite.)

$$
\begin{aligned}
v_{\mathrm{s}} & =\frac{-\left[\ln \left(1-v_{2}\right)+v_{2}+\mu v_{2}^{2}\right]}{2 V_{1} \rho_{1}\left(v_{2}{ }^{1 / 3}-v_{2} / 2\right)}, \\
v_{2} & =\frac{1}{1+\alpha \cdot \rho_{1} / \rho_{2} \gamma} \\
\rho & =M \cdot v_{\mathrm{s}}
\end{aligned}
$$

where the values applied in this work were as follows: $V_{1}, 107 /$ toluene, $25^{\circ} \mathrm{C} ; \mu, 0.43 /$ toluene-polychloroprene, $25^{\circ} \mathrm{C} ; \rho_{1}, 1.217 /$ polychloroprene, $25^{\circ} \mathrm{C} ; \rho_{2}, 0.858 /$ toluene, $25^{\circ} \mathrm{C} ; \gamma$, $1 /$ weight fraction of polymer in the sample; $M$, 88.5 /polychloroprene.

\section{Determination of Volume of Monomer Droplet}

$100 \mathrm{~cm}^{3}$ of the latex was sampled at the required time. The latex was centrifuged for 30 min at $8000 \mathrm{rpm}$ in a tube. The volume of the upper layer was measured.

\section{Determination of Surface-Tension}

Measurements were done according to ASTM 971-50, using Denui's surface-tension tester manufactured by Shimadzu Instrumental
Corp.

\section{RESULTS AND DISCUSSION}

Figure 1 shows the dependence of the volume of the monomer droplet on conversion. At lower conversions, the latex separated into two layers: upper/monomer and lower/aqueous phase. As the polymerization proceeded, the volume of the monomer decreased and the interface of two layers got vague. By extrapolation, monomer droplets were supposed to have disappeared at the conversion around $50 \%$.

Figure 2 shows the dependence of the

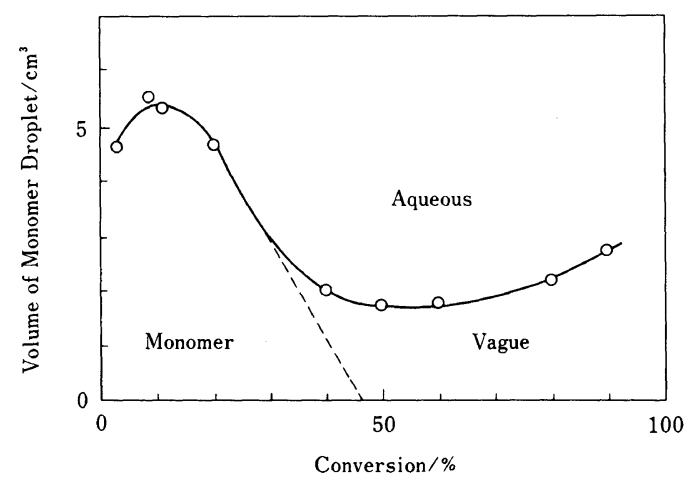

Figure 1. Variation of volume of monomer with conversion at $40^{\circ} \mathrm{C}$.

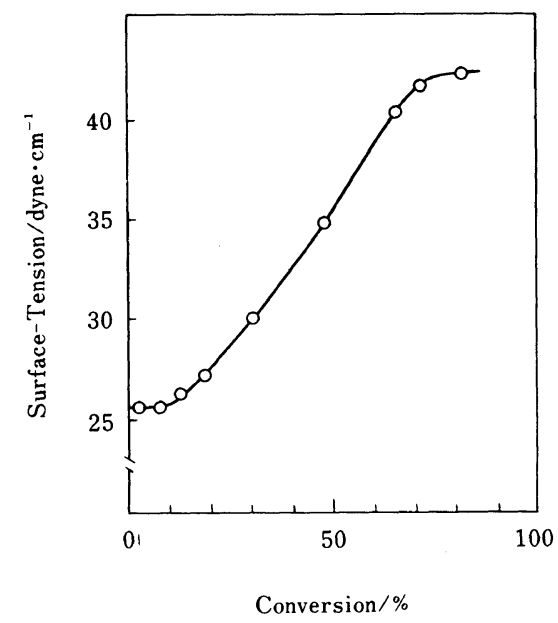

Figure 2. Variation of surface-tension with conversion at $40^{\circ} \mathrm{C}$. 


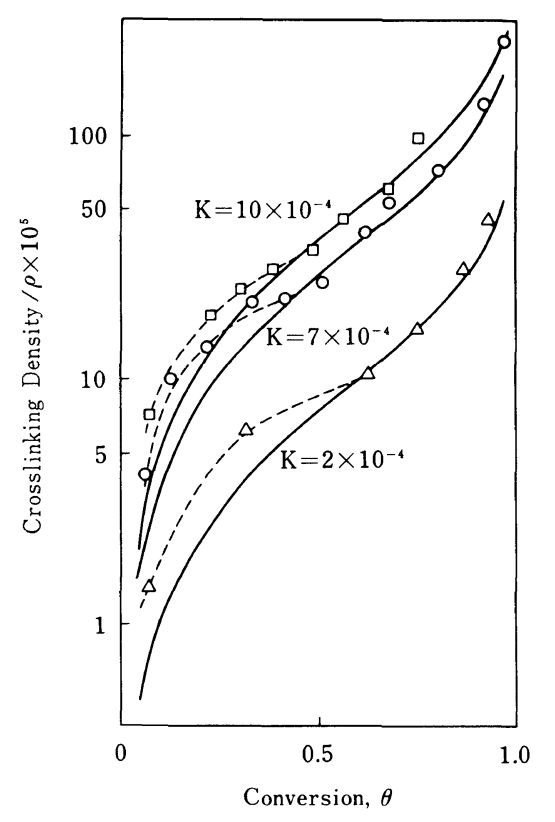

Figure 3. Variation of crosslinking density with conversion at $10^{\circ} \mathrm{C}(\triangle), 40^{\circ} \mathrm{C}(\bigcirc)$, and $55^{\circ} \mathrm{C}(\square)$. The solid curves correspond to Flory's equation at various $K$.

surface-tension on the conversion. Comparison with Harkins Model suggests that the region from 0 to $c a .10 \%$ conversion is the first stage in which the polymerization rate is increasing. The region from $c a$. 10 to $c a .50 \%$ corresponds to the second stage of constant polymerization rate, and the region of more than ca. $50 \%$ is the third stage in which the polymerization rate is proportional to the monomer content.

In Figure 3 are shown the relations between the crosslinking density and the conversion obtained at various temperatures in the absence of a chain transfer reagent. The solid curves correspond to Flory's expression (eq 1) at $K=10 \times 10^{-4}, 7 \times 10^{-4}$, and $2 \times 10^{-4}$, respectively. Above $50 \%$ conversion, experimental data coincided with those expected from Flory's equation. Below ca. $50 \%$ conversion, however, these experimental data deviated from the expected ones. This was due to an assumption that the concentration of polymer in the growing polymer particle is equal to the conversion.
We therefore tried to modify Flory's expression (eq 1) in terms of the Smith-Ewart's kinetics, ${ }^{12}$ i.e., "the ratio of polymer to monomer is constant in the growing polymer particles during the period when monomer droplets exist".

Flory ${ }^{13}$ suggested a simple treatment with a relationship of double-bonds of polymer and monomer with the growing free radical. He obtained the following expression:

$$
\mathrm{d} v / \mathrm{d} \theta=K \theta N_{0} /(1-\theta)
$$

After integrating,

$$
\begin{aligned}
v=-K & {\left[N_{0} \theta+N_{0} \ln (1-\theta)\right] } \\
& \rho=v / \theta N_{0}=-K[1+(1 / \theta) \ln (1-\theta)],
\end{aligned}
$$

where the notations are the same as in eq 1 , except for $v$ and $N_{0} ; v$ is the number of crosslinking units, and $N_{0}$ is the number of initial total units. According to the Harkins Model, the polymerization mechanism should be divided into two stages: less than and more than $\theta^{\prime}$ of $\theta$, where $\theta^{\prime}$ is the conversion at the moment of disappearance of monomer droplets.

In $0 \leqq \theta \leqq \theta^{\prime}$ the monomer droplets exist, and the proportion of polymer in the growing polymer particle is constant $\theta^{\prime}$, according to the kinetics of Smith and Ewart. Then,

$$
\begin{aligned}
& \mathrm{d} \theta / \mathrm{d} t=k_{\mathrm{p}}[\mathrm{M} \cdot]\left(1-\theta^{\prime}\right) \\
& \mathrm{d} v / \mathrm{d} t=2 k_{\mathrm{b}}[\mathrm{M} \cdot] \theta N_{0}
\end{aligned}
$$

From these two equations,

$$
\mathrm{d} v / \mathrm{d} \theta=\left(2 k_{\mathrm{b}} / k_{\mathrm{p}}\right) \theta N_{\mathrm{o}} /\left(1-\theta^{\prime}\right)
$$

After integrating eq 4 , we have

$$
v=K \cdot \frac{1}{2} \cdot \frac{1}{\left(1-\theta^{\prime}\right)} \cdot \theta^{2} N_{0}
$$

or

$$
\rho=v / N_{0} \theta=K \theta /\left[2\left(1-\theta^{\prime}\right)\right]
$$

In $\theta^{\prime} \leqq \theta \leqq 1$ the monomer droplets do not exist, all monomer and polymer molecules are located in the growing polymer particles. We 
have

$$
\begin{gathered}
v^{\prime}=K \cdot \frac{1}{2} \cdot \frac{1}{\left(1-\theta^{\prime}\right)} \cdot \theta^{\prime 2} N_{0} \\
\Delta v=\int_{\theta}^{\theta}, K \theta N_{0} /(1-\theta) \cdot \mathrm{d} \theta \\
=-K\left[\left\{N_{0} \theta+\left(N_{0} \ln (1-\theta)\right)\right\}\right. \\
\\
\left.-\left\{N_{0} \theta^{\prime}+N_{0}\left(1-\theta^{\prime}\right)\right\}\right]
\end{gathered}
$$

where $v^{\prime}$ is the number of crosslinking units at $\theta=\theta^{\prime}$, and $\Delta v$ is the increment of crosslinking units from $\theta=\theta^{\prime}$ to $\theta$. Then,

$$
\begin{aligned}
\rho & =\left(v^{\prime}+\Delta v\right) / N_{0} \theta \\
& =-K[1+(1 / \theta)\{\ln (1-\theta) / \exp (a)\}]
\end{aligned}
$$

where

$$
a=\theta^{\prime 2} / 2\left(1-\theta^{\prime}\right)+\theta^{\prime}+\ln \left(1-\theta^{\prime}\right)
$$

$\theta^{\prime}$ and $K$ were calculated at 10,40 , and $55^{\circ} \mathrm{C}$ with the aid of eq 6 and 10.

The calculation was done by a trial and error method in which the initial value of $\theta^{\prime}$ is presumed to be 0.5 . The values for conversion are used after averaged, and $\theta^{\prime}$ is calculated from the averaged $K$ and itself averaged. Then, these procedures are iterated. For values of $K$, we obtained $2.0 \times 10^{-4}, 7.1 \times 10^{-4}$, and $9.9 \times 10^{-4}$, at $\theta^{\prime}=0.47,0.45$, and 0.42 at 10 , 40 , and $55^{\circ} \mathrm{C}$, respectively.

Figure 4 shows the Arrhenius plot for $K$. The difference in activation energies between crosslinking and propagation, $\Delta E=E_{\mathrm{b}}-E_{\mathrm{p}}$, was found to be $27.8 \mathrm{~kJ} \mathrm{~mol}^{-1}\left(6.6 \mathrm{kcal} \mathrm{mol}^{-1}\right)$. This value is a little lower than that of butadiene, ${ }^{14} 7.5 \mathrm{kcal} \mathrm{mol}^{-1}$, determined by a different method.

In Figure 5 is presented a comparison between the experimental data and the curves of eq 6 and 10. The experimental data are almost identical with the theoretically predicted ones. More precisely, there are some deviations of experimental $\rho$ values from the theoretical ones, both near $\theta^{\prime}$ and at high conversions. This trend suggests that near $\theta^{\prime}$ conversions the surface concentration of the emulsifier got

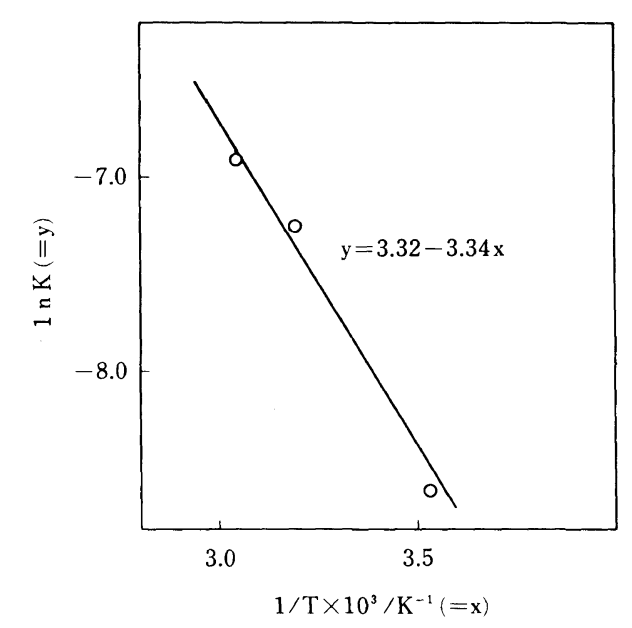

Figure 4. Arrhenius plot for $K$ to determine $\Delta E=$ $E_{\mathrm{b}}-E_{\mathrm{p}}$

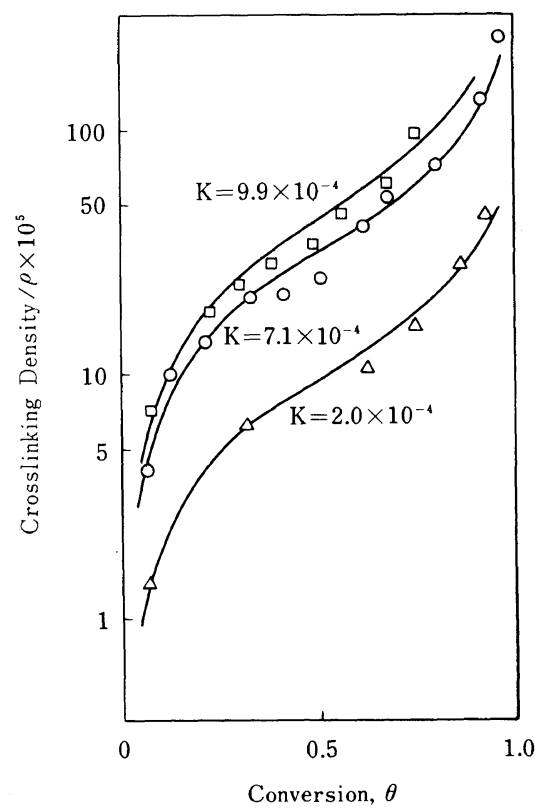

Figure 5. Variation of crosslinking density with conversion at $10^{\circ} \mathrm{C}(\triangle), 40^{\circ} \mathrm{C}(\bigcirc)$, and $55^{\circ} \mathrm{C}(\square)$. The solid curves are theoretical ones, at various $K$.

lower, and the change of the interfacial energy made the proportions of polymer in the growing polymer particles lower than the averaged value, $\theta^{\prime}$.

Acknowledgments. We would like to ex- 
press our sincere thanks to Denki Kagaku Kogyo Co., Ltd. for supporting this study and Professor Riichirô Chûjô of Tokyo Institute of Technology for his helpful discussions.

\section{REFERENCES}

1. W. H. Carothers, I. Williams, A. M. Collins, and J. E. Kirby, J. Am. Chem. Soc., 53, 4203 (1931).

2. W. E. Mochel and J. H. Peterson, J. Am. Chem. Soc., 71, 1426 (1949).

3. M. Morton, J. A. Cala, and M. W. Altier, J. Polym. Sci., 19, 545 (1956).

4. M. Morton and I. Piirma, J. Polym. Sci., 19, 563 (1956).

5. A. Rysanek, J. Polym. Sci., 29, 559 (1958).

6. Z. Manyasek and A. Rezabek, J. Polym. Sci., 56, 47 (1962).
7. J. W. Mcfarland and R. Pariser, J. Appl. Polym. Sci., 7, 675 (1963).

8. F. Hrabak, M. Bezedek, V. Hynkova, and Z. Pelzbauer, J. Polym. Sci., C-3, 16, 1345 (1967).

9. R. V. Bagdasaryan, Vysokomol. Soedin. Ser. B., 16, 858 (1974).

10. C. A. Stewart, Jr., T. Takeshita, and M. L. Coleman, "Encyclopedia of Polymer Science and Engineering," Vol. 3, "Chloroprene Polymers," John Wiley \& Sons, New York, N.Y., 1985.

11. W. D. Harkins, J. Am. Chem. Soc., 69, 1428 (1947).

12. W. V. Smith, J. Am. Chem. Soc., 68, 2059, 2064, 2069 (1946); W. V. Smith and R. H. Ewart, J. Chem. Phys., 16, 592 (1948).

13. P. J. Flory, "Principles of Polymer Chemistry," Cornell University Press, Ithaca, New York, N.Y., 1953.

14. M. Morton and P. P. Salatiello, J. Polym. Sci., 6, 225 (1951). 